Association for Information Systems AIS Electronic Library (AISeL)

Wirtschaftsinformatik Proceedings 2005

Wirtschaftsinformatik

February 2005

\title{
Integration of Electronic Foreign Exchange Trading and Corporate Treasury Systems with Web Services
}

Hong Tuan Kiet Vo

University of Karlsruhe (TH)

Christof Weinhardt

University of Karlsruhe $(\mathrm{TH})$

Remigiusz Wojciechowski

Bayer $A G$

Follow this and additional works at: http://aisel.aisnet.org/wi2005

\section{Recommended Citation}

Vo, Hong Tuan Kiet; Weinhardt, Christof; and Wojciechowski, Remigiusz, "Integration of Electronic Foreign Exchange Trading and Corporate Treasury Systems with Web Services" (2005). Wirtschaftsinformatik Proceedings 2005. 25.

http://aisel.aisnet.org/wi2005/25

This material is brought to you by the Wirtschaftsinformatik at AIS Electronic Library (AISeL). It has been accepted for inclusion in Wirtschaftsinformatik Proceedings 2005 by an authorized administrator of AIS Electronic Library (AISeL). For more information, please contact elibrary@aisnet.org. 
In: Ferstl, Otto K, u.a. (Hg) 2005. Wirtschaftsinformatik 2005: eEconomy, eGovernment, eSociety; 7. Internationale Tagung Wirtschaftsinformatik 2005. Heidelberg: Physica-Verlag

ISBN: 3-7908-1574-8

(C) Physica-Verlag Heidelberg 2005 


\title{
Integration of Electronic Foreign Exchange Trading and Corporate Treasury Systems with Web Services
}

\author{
Hong Tuan Kiet Vo, Christof Weinhardt \\ University of Karlsruhe (TH)
}

Remigiusz Wojciechowski

Bayer AG

\begin{abstract}
Hedging foreign currency risk is a central task for treasuries of multinational corporations. Traditionally, it has strongly relied on voice-based broking. With the advent of Internet technology, online platforms for foreign exchange trading emerged offering corporations a single point of access to the liquidity of a global market. However, online marketplaces only become truly valuable if seamlessly integrated into the corporation's hedging process. This paper evaluates the strengths and weaknesses of multi-bank online marketplaces, identifies the demanding and complex integration challenge and presents a Web Services-based straight-through processing implementation as a manageable approach to integrate online marketplaces and corporate treasury systems ${ }^{l}$.
\end{abstract}

Keywords: foreign exchange trading process, Web Service, Treasury Management System (TMS), voice-based broking, single-bank online marketplaces, multi-bank online marketplaces, straight through processing (STP), B2B integration

\section{Introduction}

Hedging foreign currency risk is a central task for treasuries of multinational corporations. Traditionally, it has strongly relied on voice-based broking. With the

\footnotetext{
This work reflects experiences and lessons learned from the Corporate Financial Portal project, a cooperation between the Bayer AG, the Chair for Information Management and Systems Prof. Dr. Christof Weinhardt and the Chair for General Business Administration, International Management, Accounting and Auditing Prof. Dr. Martin Glaum. The primary project objective is to identify und provide relevant financial services and information through a single point of entry within the corporate intranet. Thus there is a strong focus on application and process integration as data from various sources and channels has to be integrated.
} 
advent of Internet technology, online platforms for foreign exchange trading emerged offering corporations a single point of access to the liquidity of a global market. Better transparency and price discovery are often pointed out benefits of this trading channel [Radi01, Gtne01, Sanb02]. However, online marketplaces only become truly valuable if seamlessly integrated into the company's hedging process [Sanb02]. Such integration is a demanding and complex task as the hedging process exceeds company borders. Companies are therefore reluctant when deciding about the use of this trading channel.

The integration complexity can be reduced by employing Web Services, which promise to help overcome integration barriers as connectivity and interoperability issues are addressed within the underlying standards [Lint04, p.311]. Samtani and Sadhwani discuss the role of Web Services in the context of straight-through processing (STP) [SaSa02a] and $\mathrm{B} 2 \mathrm{~B}$ integration $[\mathrm{SaSa} 02 \mathrm{~b}]$ and conclude that the technology is appropriate to integrate heterogeneous systems and processes.

This paper evaluates multi-bank online marketplaces, identifies the complex integration challenge as the main reason for the reluctant adoption of the this trading channel and finally presents a Web Services-based straight-through processing implementation as an manageable approach to integrate online marketplaces and corporate treasury management systems.

The paper is structured as follows: section 2 focuses on foreign exchange trading. After the traditional voice-based trading process is outlined, electronic trading with multi-bank online marketplaces is addressed and evaluated in detail. Thereafter section 3 identifies the challenges and implications of straight-through processing and evaluates Web Services for integration of external systems. Based on the insights of the preceding sections, section 4 describes how electronic trading can be integrated into the corporate trading process using Web Services. The work concludes with a summary of lessons learned and an outlook identifying further research topics.

\section{Foreign exchange trading}

For multinational industrial companies, buying goods and material or selling products worldwide is part of their every day business. As a result, they have to deal with receivables and payables denominated in foreign currencies. It is common practice to hedge such items against foreign currency risk using derivative foreign exchange contracts. 


\subsection{The foreign exchange trade lifecycle}

The foreign exchange trading process can be classified into four phases $\left[\mathrm{Pic}^{+} 96\right.$, p.16]: the information phase (pre-trade phase), the order routing phase, the trade phase and finally the settlement phase (post-trade phase). Figure [1] gives an overview about the trading phases and illustrates them in the context of the involved parties.

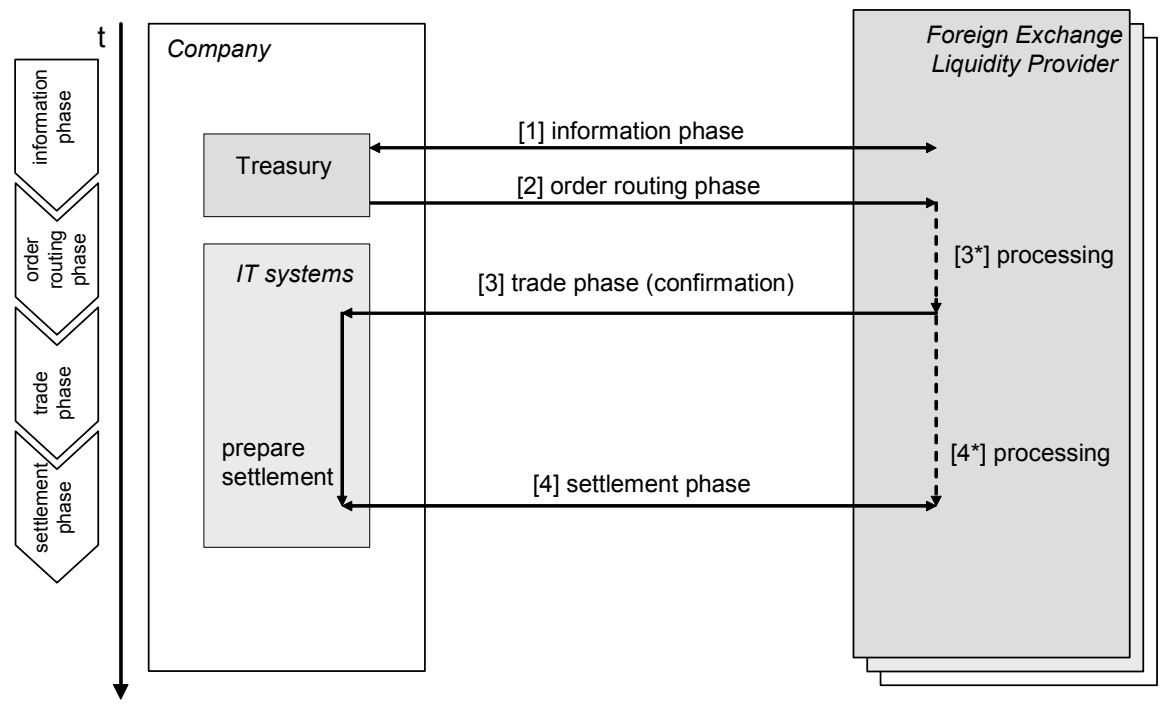

Figure 1: Foreign exchange trading process

The information phase [1] involves determining the parameters of the required foreign exchange deal (e.g. contract type, volume, maturity etc.) and identifying possible liquidity providers (i.e. sell-side counterparties). During the subsequent order routing phase [2], requests for quote are processed to determine matching offers. As soon as an offer has been accepted, the foreign exchange contract is closed and confirmed within the trade phase [3]. Final settlement and clearing procedures are prepared and executed during the settlement phase [4].

This work focuses on the corporate perspective of the foreign exchange trading process therefore phases [3*] and [4*] are not discussed. 


\subsection{Voice-based foreign exchange trading}

Traditionally, the task of dealing in foreign exchange has been accomplished using voice-based broking. The corporate dealer calls one or more ${ }^{2}$ counterparty banks, which serve as foreign exchange liquidity providers. During each phone call the dealer specifies the key parameters of the contract (e.g. currency pair, amount and maturity) and requests a corresponding quote. Once a sufficient number of quotes is available, the dealer chooses the best quote. Figure 2 illustrates this process.

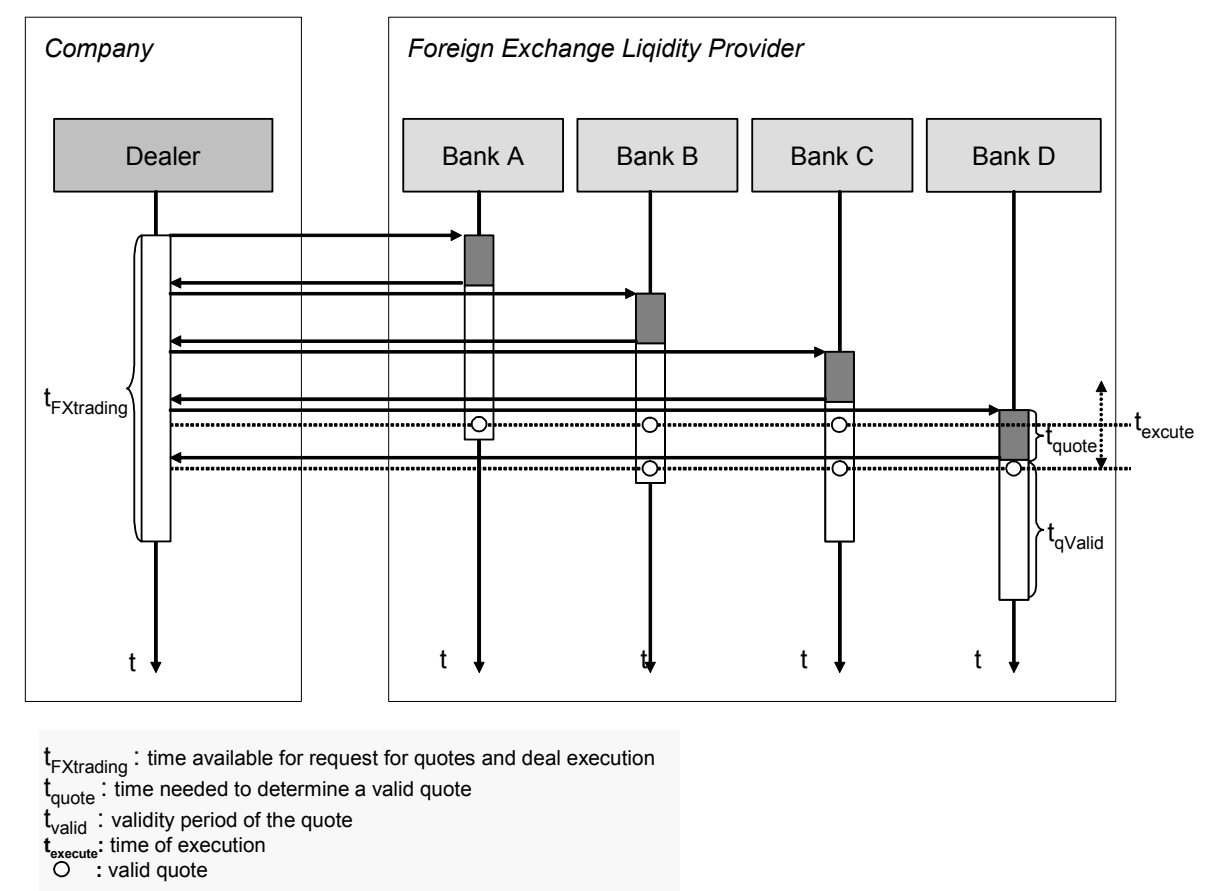

Figure 2: Voice-based foreign exchange trading process

Obviously, the major drawback of voice-based broking is that each request for quote has to be executed strictly sequentially due to the physical limitations of the medium $^{3}$. Moreover, since offered quotes are valid for only a limited time $\left(t_{\mathrm{qValid}}\right)$ the maximum number of valid quotes that the corporate dealer can request is limited [Radi01]. In the situation illustrated in Figure [2], it is impossible for the dealer to obtain more than three valid quotes from different liquidity providers at

Usually internal guidelines prescribe competitive bidding thus compelling foreign exchange dealers to contact more than one counterparty.

3 This drawback can only be overcome by introducing more traders, who trade simultaneously. 
the same time, because as soon as the fourth counterparty is contacted the quote from the first will expire.

Furthermore, the fact that information exchanged over the phone needs to be manually entered into the treasury management system (TMS) makes this process error prone.

\subsection{Electronic foreign exchange trading}

Internet technology paved the way from aforementioned voice-based broking to online platforms for trading in foreign exchange. Single-bank online platforms, which appeared at first, were followed by multi-bank marketplaces ${ }^{4}$.

\subsubsection{Single-bank online platforms}

Single-bank online marketplaces enable customers to access liquidity and services offered by a single bank through the Internet. However the mere replacement of telephone by a web browser only represents a change in the trading channel without helping overcome the limitations of voice-based trading mentioned in the previous section. On the contrary, the situation deteriorates as the corporate dealer has to get accustomed to use different web-based trading systems, which often results in an increased amount of time needed to retrieve quotes as well as an higher probability for producing manual errors due to necessary data re-keying.

Nevertheless, single-bank platforms as a electronic medium can be connected with corporate treasury management system solving the above mentioned drawback. Figure [3] illustrates the situation where different single-bank marketplaces are integrated into the TMS using custom built interfaces.

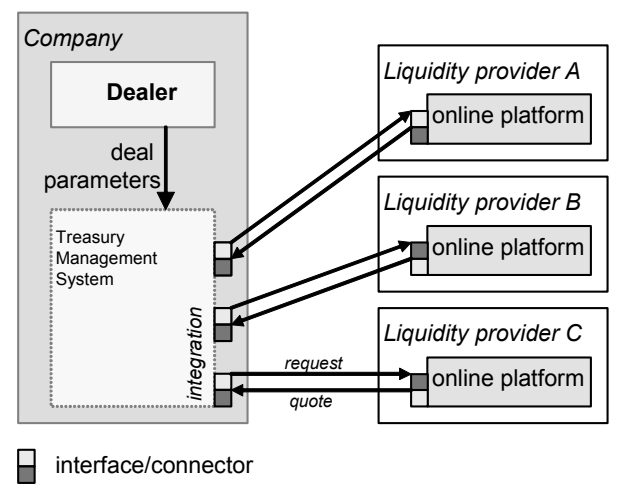

Figure 3: Integration of single-bank trading platforms and corporate TMS

4 [Kölz01] gives a comprehensive overview of e-trading platforms. 
Having integrated the liquidity providers, the corporate dealer now only needs to specify the trade details once. The TMS then connects to the different available counterparties, requests quotes and provides the results virtually simultaneously to the dealer.

However, since each bank maintains its own platform, the company will usually have to integrate $n$ systems for $n$ counterparties. Building and particularly maintaining interfaces is a very costly procedure [Lint00]. Therefore in reality the number of different liquidity providers integrated and thus available is bound to be limited.

Furthermore, from a global perspective, if $m$ companies need to integrate $n$ liquidity providers, $m^{*} n$ interfaces need to be built in order to establish a network ${ }^{5}$ (see Figure [4]). This inefficiency can be dealt with through the introduction of an intermediator, which offers system integration as a service and reduces the number of needed interfaces to $m+n$. This demand is one of the main drivers behind the introduction of multi-bank online marketplaces.

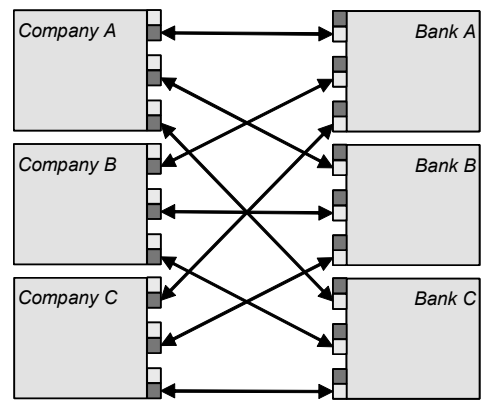

Figure 4: Benefits of intermediation

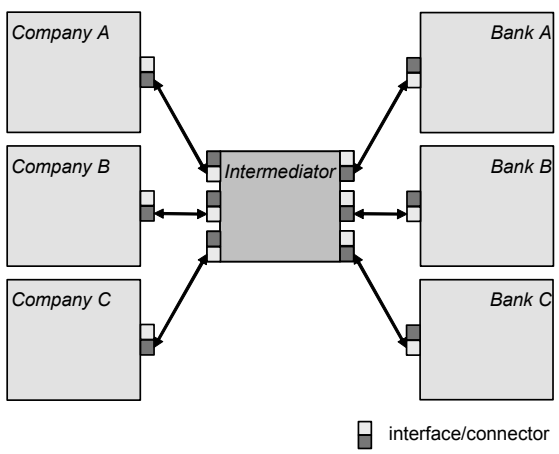

日 interface/connector

\subsubsection{Multi-bank online marketplaces}

Multi-bank online marketplaces offer corporate costumers access to their relationship banks by integrating automated pricing services provided by the banks [Sanb02]. Corporate costumers can request live, executable quotes simultaneously from their counterparties using this trading channel. The number of quotes is limited by the counterparty's response time, technical constraints as well as the number of available sell-side counterparties. Figure [5] illustrates the trading process.

5 The situation reflects the negative effect of "Metcalfe's Law" [ShVa98] 


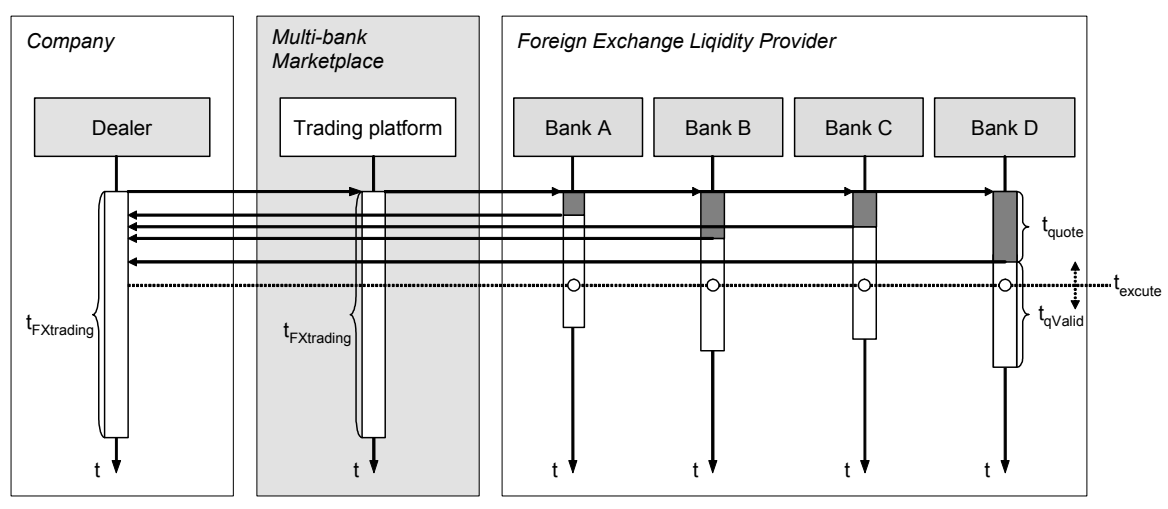

$t_{F X \text { trading: time available for request for quotes and deal execution }}$

$t_{\text {quote: }}$ : time needed to determine a valid quote

$\mathrm{t}_{\mathrm{vali}}$ : validity period of the quote

$t_{\text {execute }}$ : time of execution

Figure 5: FX trading process with multi-bank online marketplace

After executing the request for quote the FX marketplace forwards the request to the pricing services of the chosen liquidity providers. After the request is processed $\left(\mathrm{t}_{\text {quote }}\right)$, the pricing service will respond a valid executable price, which is forwarded to the corporate dealer in real time. Usually the dealer has to confirm an FX deal within a certain fixed time span $t_{\mathrm{FXtrading}}$ thus quotes taking longer to respond will not be available and therefore lowering the number of theoretically available quotes. Finally the dealer compares the obtained competitive bids and chooses the one that fits his needs best while considering $\mathrm{t}_{\mathrm{qValid}}$.

\subsection{Evaluation of electronic FX trading and multi-bank marketplaces}

Multi-bank marketplaces from an intermediator perspective add value to the buyside and the sell-side customer through coordination services [Sak $\left.{ }^{+} 95\right]$. By integrating the liquidity providers, the multi-bank marketplaces not only help overcome the compatibility issue of connecting the heterogeneous systems [Gomb01] but more importantly offer the corporate customer access to a single foreign exchange market with the accumulated liquidity of the participating relationship banks. Liquid markets are considered less volatile than illiquid ones [Harr03, p. 394]; consequently immediate trading of high volume deals without significantly affecting the market price is possible [O'Har97].

Using voice-based broking, it is very difficult if not virtually impossible to get an idea about the real market price of a transaction. Multi-bank marketplaces on the 
other hand allow the corporate dealer to observe and compare multiple competitive bids simultaneously therefore supporting price discovery as well as better market transparency [Radi01].

The competitive bids can be recorded with further trade information to build a solid data basis for data mining and post trade analysis [Radi01]. Mining data on trade details like bids, spreads etc. can provide answers to various questions and can, e.g. become useful when negotiating with relationship banks concerning general business conditions.

However, multi-bank marketplaces are not the holy grail of foreign exchange trading. The relationship banks actually have no motivation to join such platforms as they benefit from corporate business in the traditional state.

The lack of personal relationships is another aspect often mentioned in the context of multi-bank marketplaces [Sale02]. Personal relationships are often believed to result in faster and better quotes especially when trading high volume deals [Glob01]. Together with the fear of giving away precious business information to competitive market participants when searching for counterparties to settle high volume deals, these are important reasons for companies to limit their online FX trading to lower volume deals only [Gtne02].

Increased operational efficiency and control often regarded as the central benefit of electronic trading [Sanb02] is a double-edged sword. Although companies can improve their FX trading process with the help of electronic trading, the integration challenge is far from being trivial and is therefore considered as a major hurdle for the adoption of this trading channel [Sale02]. Operational efficiency is hardly improved by merely changing the trading medium from voice-based broking to electronic trading. In order to achieve significant cost savings it is necessary to seamlessly integrate the new process into the company's entire foreign exchange trading process. Although major multi-bank marketplace vendors support integration by providing interfaces for commonly deployed treasury management systems, the task to integrate the heterogeneous systems remains and there is always the possibility, that the interfaces provided do not fit to the actual treasury system.

Furthermore, electronic multi-bank marketplaces services do not encompass trade confirmation and matching. Traditionally, trade confirmation and matching has been performed manually and involves the generation of confirmation letters. As the number of possible counterparties increases with multi-bank marketplaces, companies will have to make sure that they are still able to integrate them into their trade confirmation process ${ }^{6}$.

6 Confirmation matching service providers (e.g. Misys - www.misys.com) offer an solution to this dilemma, as they integrate the different liquidity providers and offer a single point of access to confirmation and matching information. 


\section{Straight-through processing and Web Services}

\subsection{Straight-through processing in the financial industry}

\subsubsection{Straight-through processing and integration}

The term straight-through processing (STP) is used in the financial industry in the context of achieving end-to-end processing of financial transaction from initiation to settlement [SaSa02a] through the automation of financial (sub-)processes. Ideally a manual interaction is only required at the start of a transaction while subsequent phases are processed without the need for any further human interferences. Consequently the risk of possible additional manual errors i.e. through mis-keying trade details or through delays is eliminated [Smith03]. In order to achieve this goal heterogeneous information systems that are involved in financial transactions have to be seamlessly connected, often alongside with the redesign of business processes [SaSa02a]. Thus STP is about solving the question of how to integrate these systems [Lint04] while keeping integration itself manageable (i.e. at low cost) - a complex task, as external and internal integration problems have to be solved [Wei $\left.{ }^{+} 03\right]$.

Internal STP refers to streamlining financial processes that are internal to an industry participant [SaSa02a], a topic that is discussed in the context of enterprise application integration (EAI). External STP on the other hand addresses the challenge of seamlessly integrating external partners that are involved in financial processes [SaSa02a]. Therefore cross company coordination, security of data exchange as well as system connectivity and interoperability are key issues that have to be considered [FiCo01]. External STP is discussed within the context of B2B integration ${ }^{7}$.

\subsubsection{Evaluating the consequences of process automation}

The importance of STP for the financial industry can be compared to the role supply chain management plays for the manufacturing industry or customer relationship management for the service industry [SaSa02a]. Important drivers pushing STP initiatives are [Eldr04, SaSa02a, FiCo01, Tayl03]:

\footnotetext{
Weitzel $\left[\mathrm{Wei}^{+} 03\right]$ analyses coordination in the context of B2B integration resulting in the need for industry-wide standards, whereas this work will concentrate on the technological aspects of connecting the heterogeneous systems across company borders.
} 
- The elimination of unnecessary manual interactions reduces the number of errors consequently increasing efficiency and reducing operational risk.

- Financial transactions can be performed faster and a higher number of transactions can be processed without a decrease in the quality of a single transaction.

- Changes in financial strategies can be performed faster and more accurate.

- Increased operational efficiency and time savings reduce operational cost.

Despite the above stated benefits, process automation also bears risks that must not be overlooked. With ongoing process streamlining, manual interventions will be reduced to a minimum alongside with errors. But manual interventions also serve as sanity checkpoints in case data is erroneous. Humans will usually at least skip transactions as soon as they notice possible errors or simply do not understand instructions. Automated processing on the other hand must include methods that validate data and detect possible errors right from the start. Also exception handling routines must be implemented in case of errors; otherwise there is the risk that unrecognized errors might ultimately result in a cascading amount of faulty transactions [Eldr04]. Consequently a possibility to interrupt a process must be implemented.

Automation hides details about the underlying processes as well as the involved procedures executed in the background. Consequently there is the possibility that people forget about the details behind the automated process. This will prove troublesome in case of business exceptions i.e., errors, when automation fails and transactions have to be performed manually again.

As a consequence STP initiatives must consider not only the potential benefits but also the possible threats of process automation avoiding poorly designed STP initiatives. Critical parameters, especially from a technological perspective, that determine the success or failure of STP initiatives are transaction speed, accuracy of trade information, overall stability and extensibility of the chosen technological infrastructure as well as the level of security provided for data exchange [SaSa02a].

\subsection{Straight-through processing with Web Services}

\subsubsection{Enterprise application integration and Web Services}

Enterprise application integration seeks for methods and tools that help to connect applications, share services and data within and across company borders and networks [SaSa02b]. Concepts supporting internal and external system integration namely the Common Object Request Broker Architecture (CORBA), the Distributed Component Object Model (DCOM) or the of Remote Method Invocations 
(RMI) [WoWe02, p.102] have existed for a while however the notion of Web Services is the only one people are paying attention to today [Lint04, p. 324].

Web Services are encapsulated, loosely coupled, contracted functions offered via standard protocols that can be used to share application functionality and data over networks such as the Internet [SaVo04]. Web Services are based on established standards including XML, SOAP, and WSDL [WoWe02, p. 100] which provide remote procedure call (RPC) mechanisms that can pass through company firewalls [Maie02b]. Being operating system and programming language agnostic, Web Services can help to efficiently integrate technical heterogeneous and disparate environments [W3C03]

\subsubsection{Evaluation of Web Services and system integration}

Web Services present a paradigm shift in the way software assets can be shared and managed. Though integration via distributed systems, namely Remote Procedure Calls (RPC), is not a new idea, the notion of Web Services enriches this concept by adding the following facets [Glas00]:

- Interoperability: In contrast to existing distributed architectures, the use of Web Services is independent from the operating system and the programming language. This is backed up by the fact that vendors could agree on the proposed Web Service standards [Lint04, p. 311].

- Ubiquitous Use: Using XML to marshal data into request and respond documents and HTTP, Web Services can be virtually used by any device supporting these industry standards [Cohe02].

- Low entry-barriers: simple concepts behind Web Services make it easy to adopt this technology (also from a cost point of view).

- Wide acceptance throughout the software industry: support from companies like Microsoft, Sun, IBM, SAP, etc. [SmBa01].

Nevertheless there exist unresolved issues concerning the use of Web Services that are discussed and should be therefore considered when evaluating the application of Web Services [Smit01].

Trade information are highly business relevant data thus security must be considered when using Web Services to transfer and consume the precious information. Web Service performance is another critical factor that must be evaluated. Although the number of service requests from a single company might be relatively low, the service will most likely not be consumed by a single party only thus the absolute number of request that must be handled might be very high. The per-

8 Wojciechowski [WoWe02] gives a comprehensive overview about the Web Service standard. 
formance of Web Services is highly dependent on its implementation - poor implementation can highly affect the overall service scalability (i.e. performance) [Lito02].

Finally Web Services still have to be integrated into existing IT systems. Interface and service will have to be build that consume the service and transform the data into a format that is required by the underlying IT systems. Integration using Web Services still is not an out-of-the-box event [Lint03].

\section{Integration of electronic foreign exchange trading and corporate treasury systems with Web Services}

The following section will present lessons learned from implementing a straight through processing initiative at Bayer AG Corporate Finance. The objective was to streamline and enhance the corporate foreign exchange trading process with electronic trading using multi-bank marketplace and automated deal confirmation and settlement services [cp. Section 2.1].

\subsection{Integration analysis of the initial situation}

The initial situation of the company's foreign exchange trading process can be described as follows: after the corporate dealer has entered into a foreign exchange contract [1], she has to reenter the exact trade information into the company's own treasury management system [2]. The back-office staff than has to manually track confirmation of open foreign exchange deals [3] by observing data-feeds from the confirmation and matching provider [4]. In case there is a valid match and an open deal has been confirmed, the correspondent trade information is manually updated. Figure 6 gives an overview of the situation as of before integration. 


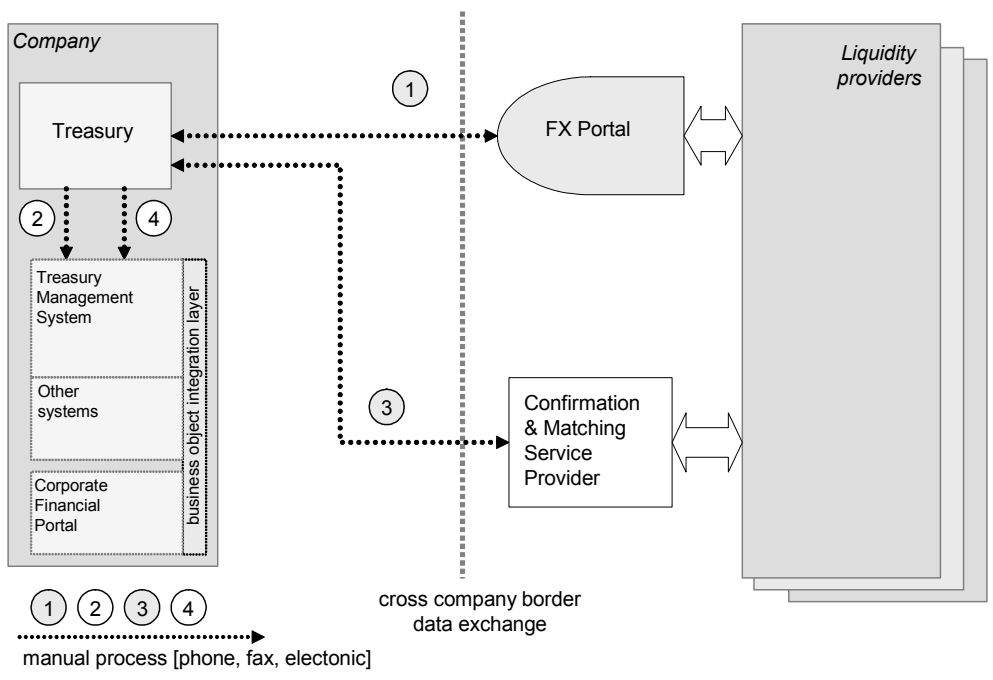

Figure 6: FX trading initial situation

\subsection{Integration implementation of electronic FX trading using Web Services}

The first step was to eliminate the need to manually reenter the deal data into the corporrate TMS after the deal has been executed using the online marketplace (cp. step [2] in figure [6]). This was achieved by connecting to the electronic trading platform to first obtain and subsequently save the deal information to the TMS (cp. [2] in Figure [7]). Usually the trading platforms provide interfaces to support the data exchange and integration. As mentioned in section [2.4] these interfaces can be based on proprietary communication protocol standards and thus often require the company to open further ports of the corporate firewall. Web Services do not suffer from this restrictions consequently integration complexity could be reduced if a service based on the Web Service standards is used.

In our specific case, the integration-service of the chosen trading platform was not based on the Web Service standard but shared important characteristics of Web Services mainly the use of standard web protocolls for communication as well as a data format based on the XML standard. As a consequence in order to consume the service, no additional ports of the corporate firewall had to be opened and the data received could be parsed and processed using standard XML tools and the semantic data description provided by the trading platform provider.

As a matter of fact the main integration hurdles turned out to be not about consuming and integrating the service but about providing a generic possibility to extract, modify and enter trade data into the corporate treasury management systems. 
Thus a lot of effort was spend on designing and building an integration layer that offered interfaces, functions and common business objects to encapsulate, transform and process the business data. This layer was implemented using Microsoft .Net technology which was chosen because of its native support for XML and Web Services and due to already existing experiences using this technology in similar projects.

Designing and implementation of the integration layer required aproximately twelve man-days with a significant amount of this time being spend on designing a highly reusable object oriented architecture. Using this integration layer a service was designed and implemented which periodically (every 15 minutes) consumes the trading platform's integration service to check for new deals, parses the XML data received from the service, maps the information to the common trade object provided by the integration layer and finally saves the extracted information to the corporate TMS.

Manual deal matching and confirmation (cp. step [3] and [4] in figure 6) was eliminated by integrating the trade confirmation process using the integrationservice offered by the CMS provider. The implementation execution was similar to the integration of the online trading platform service described above. Again a service was build that periodically consumes the CMS integration-service to check for newly confirmed trades, extracts this information and uses business objects and interfaces from the integration layer described above to save the information to the TMS. Implementation of this service could be carried out significantly faster as the integration layer could be highly reused.

In summary it took the team approximately sixteen man-days to implement the straight through processing initiative. The streamlined corporate foreign exchange trading process is illustrated in Figure [7]. 


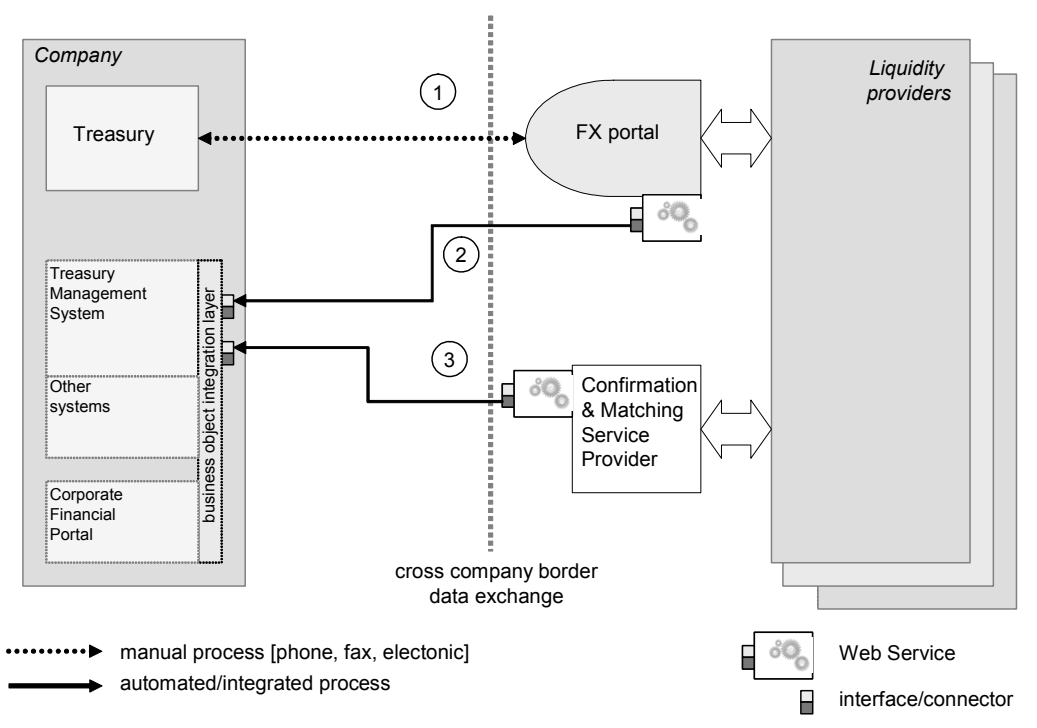

Figure 7: Integrated FX trading process

\section{Conclusion and further work}

This paper evaluates electronic foreign exchange trading with multi-bank online marketplaces. Through this trading channel the corporate dealer gains access to the liquidity of a global market rather than fragmented single markets and benefits from better transparency and price discovery. However the prospect of achieving higher operational efficiency when seamlessly integrating electronic trading into the corporate trading process reflects the true motivation behind the adoption of this trading channel. The integration complexity can be reduced by employing Web Services which help solving connectivity and interoperability issues when integrating external systems. This paper therefore describes a Web Services-based straight-through processing implementation as a manageable approach to integrate online marketplaces and corporate treasury systems to realize an efficient and streamlined FX trading process.

Next steps are to determine the ratio of deals executed using the electronic trading platform versus traditional trading channels (STP-rate), to evaluate how this ratio changes over time, to identify aspects that have an effect on the STP-rate and to quantify the operational cost savings due to the STP implementation. 


\section{References}

[Blai02] Blair, D.: Grasping the Nettle: Reaping eFX Benefits Now and in the Future, GTNews, 2002, http://www.gtnews.com/article/4788.cfm (03. July 2004)

[Cohe02] Cohen, F.: Understanding Web service interoperability, IBM developerWorks, 2002, http://www-106.ibm.com/developerworks/webservices/library/ws-inter.html (28.Juni 2004)

[Eldr04] Eldridge, G.:Deal Execution in an STP World - Part 2: Front/Middle Office Risks, GTNews 2004, http://www.gtnews.com/article/5330.cfm (11. June 2004)

[FiCo01] First Consulting: A Train called "Straight Through Processing", 2001, http://perso.wanadoo.fr/first-consulting/Letters/Letter3.pdf, (27. June 2004)

[Glas00] Glass, G.: The Web services (r)evolution - applying Web services to applications, 2000, http://www-106.ibm.com/developerworks/library/ws-peer1.html (11. June 2004)

[Glob01] Globalinvestormagazine: Foreign Exchange Survey, Globalinvestormagazine, March 2001, http://www.globalinvestormagazine.com/pdf/GIMarch01.pdf (11. July 2004)

[Gomb99] Gomber, P.: Elektronische Handelssysteme, Physica-Verlag Heidelberg, 1999

[Gtne01] GTNews: Multi-Bank FX Platforms line up to bring cost savings to treasurers, GTNews, 2001, http://www.gtnews.com/article/3916.cfm (11. June 2004)

[Gtne02] GTNews: What do corporates want from their banks? GTNews, 2002, http://www.gtnews.com/article/4794.cfm (11. July 2004)

[Gtne03] GTNews: Delivering the promise, GTNews, 2003, http://www.gtnews.com/article /4892.cfm (03. July 2004)

[Harr03] Harris, L.E.: Trading and Exchanges, Oxford, 2003, Oxford University Press

[O'Ha97] O’Hara, M.: Market Microstructure Theorie, Blackwell Publishers, 1997

[Kölz01] Kölzer, C.: E -Trading Platforms, GTNews, 2001, http://www.gtnews.com/ article/3399.cfm, (10. July 2004)

[Kuts02] Kutschke, G.: Trading Forex and Money Markets via the Internet, GTNews, 2002, http://www.gtnews.com/article/4476.cfm (5. July 2004)

[Lito02] Litoiu, M.: Migrating to Web Services - Latency and Scalability, in: Proceedings Fourth International Workshop on Web Site Evolution, 2002, p.13-20

[Lint00] Linthicum, D. S.: Enterprise Application Integration, London 2000

[Lint04] Linthicum, D.: Next Generation Application Integration - From Simple Information to Web Services, Addison-Wesley 2004

[Maie02a] Maier, G. E.: Web Services for STP, Back Office Focus, May 2002

[Maie02b] Maier, G. E.: Straight Through Processing for the Masses, Trading Technology Week, Vol. 5/27, May 2002 
[Meye03] Meyer, O.: Integration - the key to back office competitiveness, Back Office Focus, 2003, http://www.isg-alumni.com/diplomes_une/creations/informations_etps/ omeyer020903.pdf (27.June 2004)

[Misy04] Mysis: Misys Wholesale Banking Systems and Currenex sign strategic partnership agreement, Misys Press Release, 2004, http://www.misys.com/mys/ media/press/pr_banking/2004/2004-05-17ba/ (9. July 2004)

[Mire00] Mirek, L.P.: The internet: automating and improving global FX, The Treasurer, Oct. 2000

[Mois01] Moiseiwitch, J.: Online Forex: Promises, Promises, CFO.com, 2001, http://www.cfo.com/printarticle/0,5317,5338|A,00.html?f=options (03. July 2004)

[Pic $\left.{ }^{+} 96\right]$ Picot A., Bortenlänger C., Röhrl H.: Börsen im Wandel - Der Einfluß von Informationstechnik und Wettbewerb auf die Organisation von Wertpapiermärkten, Fritz Knapp Verlag, Frankfurt, 1996

[Radi01] Radin, M.: The treasury and electronic trading, GTNews/Fina, 2001, http://www.gtnews.com/article/2815.cfm (27. June 2004)

[RiSo03] Richmond Software: Straight Through Processing in a Corporate Treasury, Richmond Software TMI Report, 2003, http://www.treasury-management.com /supplement/03stp/richmond.pdf (27.June 2004)

[Sak ${ }^{+}$95] Sakar, M.B., Butler, B., Steinfield, C.: Intermediaries and Cybermediaries: A Continuing Role for Mediating Players in the Electronic Marketplaces, Journal of Computer Mediated Communication, Vol.1(3), 1995

[Sale02] Sales, R.: Electronic FX: Reality or just a smoke screen, Wall Street \& Technology Online, 2002, http://www.wallstreetandtech.com/printableArticle? doc_id=WST20020306S0004 (10. June 2004)

[Sanb02] Sanborn, T.: Hype or Reality? The Hard Facts About Electronic Trading, GTNews, 2002, http://www.gtnews.com/article/4768.cfm (10. July 2004)

[SaSa02a] Samtani, G., Sadhwani, D.: Web Services and Straight Through Processing Web Services in the Financial Industry, Web Service Architekt 2002, (07. June 2004)

[SaSa02b] Samtani, G., Sadhwani, D.: B2Bi and Web Services - an intimating task? Web Service Architekt 2002, http:// (07. June 2004)

[SaVo04] Sandrock, J., Vo, .K.: E-Learning community integration with Web Services, in Multikonferenz Wirtschaftinformatik (MKWI) 2004 Essen, Band 1, p. 72 - 83

[ShVa98] Shapiro, C., Varian, H.R.: Information rules, A strategic guide to network economy, Boston, Massachusetts 1998

[SmBa01] Smith, D., Batchelder, R.: Gartner Predicts 2002: Internet Platforms and Web Services, Gartner 2001

[Smith03] Smith, J.: Online Trading: A Hands-Off Approach, GTNews Article, 2003, http://www.gtnews.com/article/4968.cfm (27.June 2004) 
[Smith03b] Smith, J.: On the move: The challenges of online trading, GTNews, 2003, http://www.gtnews.com/article/5000.cfm (27. June 2004)

[Tayl03] Taylor, G.: Why aren't treasurers buying inte eFX?, GTNews, 2003, http://www.gtnews.com/article/4999.cfm (03. July 2004)

[W3C03] W3C: Simple Object Access Protocol, 2003, http://www.w3.org/TR/SOAP/, (11 June 2004)

[WeKö03] Weitzel, T., König, W.: Netzeffekte im E-Business, in: Proceedings of the 6. Internationale Tagung Wirtschaftsinformatik 2003 (WI 2003); Dresden

[Wei ${ }^{+}$03] Weitzel, T.,Martin, S.,König, W.: Straight Through Processing auf XML-Basis im Wertpapiergeschäft, in: WIRTSCHAFTSINFORMATIK 45 (2003) 4, p.409-420

[WoWe02] Wojciechowski, R., Weinhardt C. Web Services und Peer-to-Peer Netzwerke, in Schoder, D. et al: Peer-to-Peer, Heidelberg 2002. Springer: P.99-117 